\title{
DIE THEORETISCHEN GRUNDLAGEN DES OPTISCHEN TESTS NAGH O. WARBURG
}

\author{
O. BALGKe \\ C.F. Boehringer-Soehne GMBH., Mannheim-Waldhof, \\ Deutsche Bundesrepublik
}

Eine charakteristische Eigenschaft der Enzyme ist deren Substrat- und Wirkungsspezifität. Der Katalysator Enzym arbeitet nach einem Auswahlprinzip und "entscheidet", welche Substrate umgesetzt werden, und welche der zahlreichen thermodynamisch möglichen Umwandlungen des Substrats abläuft. Diese spezifischen Eigenschaften der Enzyme bieten sich direkt als analytisches Hilfsmittel an, und es ist daher nicht verwunderlich, daß man schon in den Anfängen der Enzymchemie den Wert der enzymatischen Analyse erkannte.

Ein Beispiel soll veranschaulichen, wie man mit Hilfe der spezifischen Enzyme in einem Gemisch chemisch sehr nahe verwandter Substanzen jede einzelne Substanz quantitativ ohne zeitraubende Trennungs-Verfahren analysieren kann. Nehmen wir an, ein Gemisch drei verschiedener Ketosäuren vorliegen zu haben: Brenztraubensäure, Oxalessigsäure und $a$-Ketoglutarsäure.

Die chemische Analyse dieser drei Säuren kannz.B. so durchgeführtwerden, daß man die Säuren mit dem Carbonyl-Reagenz Phenylhydrazin umsetzt, die Hydrazone sorgfältig isoliert, fraktioniert kristallisiert, schließlich durch den Schmelzpunkt identifiziert und ihre Menge durch Wägung bestimmt. Diese Arbeitsweise erfordert sehr viel Zeit und birgt manche Fehlerquellen in sich.

Viel eleganter, schneller und sicherer, und vor allen Dingen spezifischer bestimmt man diese Säuren mit einer enzymatischen Analysenmethode. Für die Hydrierung dieser Ketosäuren stehen wasserstoffübertragende Enzyme zur Verfügung, die als wirksame Gruppe Diphospho-pyridinnucleotid enthalten: Die Lactat-Dehydrogenase (LDH), die Malat-Dehydrogenase $(\mathrm{MDH})$ und die Glutamat-Dehydrogenase (GLDH).

Jedes der genannten Enzyme ist nur spezifisch für sein Substrat: LDH für Brenztraubensäure, $\mathrm{MDH}$ für Oxalessigsäure und GLDH für $\alpha$-Ketoglutarsäure.

In einer Photometer-Küvette bestimmt man mit Hilfe dieser Enzyme in wenigen Minuten die drei Ketosäuren.

Die enzymkatalysierten Reaktionen sind, wie alle chemischen Reaktionen, Gleichgewichtsreaktionen, d.h., die eben erwähnten Dehydrogenasen vermögen die Hydrierungs-, sowie die umgekehrte Reaktion, die Dehydrierungsreaktion zu katalysieren. Die Namen der genannten Dehydrogenasen wurden also von den umgekehrten Reaktionen, den Dehydrierungsreaktionen, abgeleitet. Z.B. Lactat-Dehydrogenase von der Fähigkeit,

P.A.c. (3) -7 


\section{O. BALGKE}

Milchsäure zu Brenztraubensàure zu dehydrieren, Malat-Dehydrogenase von der Fähigkeit, Äpfelsäure zu Oxalessigsäure zu dehydrieren.

Die Anwendung der Dehydrogenasen ist allerdings nur ein Teilgebiet der enzymatischen Analyse.

O. Warburg und Mitarbeiter beschrieben 1935 eine optische Bestimmungsmethode von physiologischen Substanzen mit Dehydrogenasen, die mit Diphospho-pyridin-nucleotid $\left(\mathrm{DPN}^{+}\right)$bzw. Dihydrophospho-pyridinnucleotid (DPNH) als Coenzym reagieren. Wie wir im folgenden sehen werden, ist der Übergang des Coenzyms $\mathrm{DPN}^{+}$in DPNH und umgekehrt die dem optischen Test zugrunde liegende Reaktion. Betrachten wir zunächst die chemische Strukturformel des $\mathrm{DPN}^{+}$:

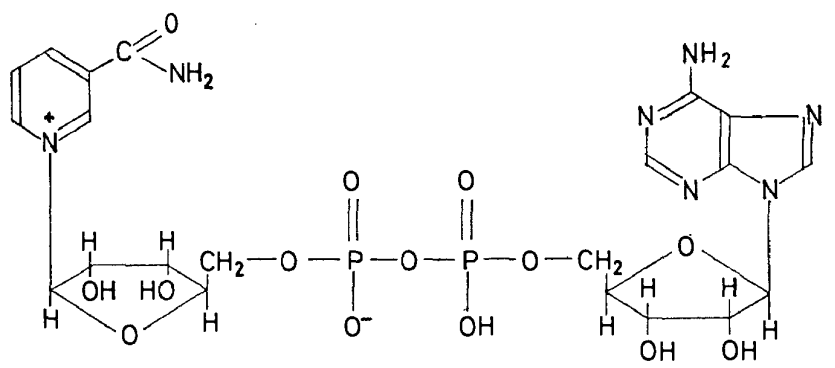

Die einzelnen Bausteine von $\mathrm{DPN}^{+}$sind Nikotinsäureamid mit dem Pyridingerüst, Ribose, Pyrophosphorsäure und Adenin. Wir finden in dem Molekül das Adenosin-5'-monophosphat, die sog. Muskel-Adenylsäure wieder, sowie das als Vitamin bekannte Nikotinsäureamid.

Der Pyridinring nimmt bei der enzymatischen Dehydrierung des Substrats einer Dehydrogenase den Substratwasserstoff auf oder gibt ihn bei der Hydrierung eines Substrats wieder ab. Diese reversible Funktion des Co-enzyms zeigt das folgende Bild:

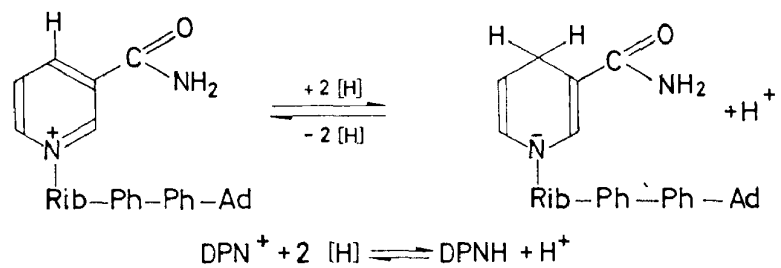

Sie sehen, daß bei der Hydrierung des $\mathrm{DPN}^{+}$die aromatische Natur des Pyridinringes aufgehoben wird. Hiermit ist eine charakteristiscle Änderung der Lichtabsorption verbunden $(A b b .1)$.

Das Dihydropyridin-System im DPNH besitzt ein breites Absorptionsmaximum bei $340 \mathrm{~m} \mu$, während das Pyridinsystem im $\mathrm{DPN}^{+}$hier nicht absorbiert. Die Adeninkomponente im $\mathrm{DPN}^{+}$und DPNH absorbiert bei $260 \mathrm{~m} \mu$.

Der Reaktionsmechanismus der Wasserstoffanlagerung an den Pyridinring des $\mathrm{DPN}^{+}$ist eingehend untersucht und sichergestellt worden. 
DIE THEORIE DES OPTISGHEN TESTS NAGH O. WARBURG

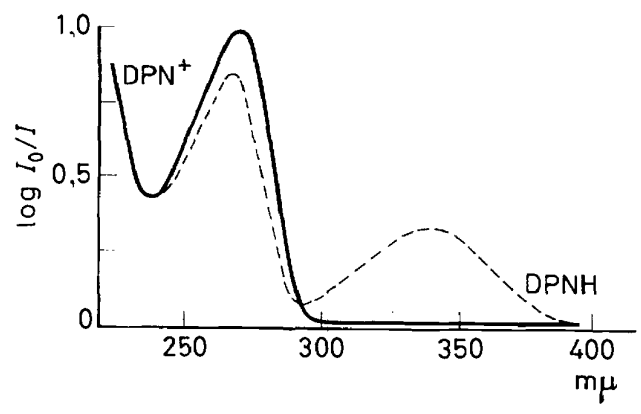

Abb. 1. Lichtabsorption von $\mathrm{DPN}^{+}$und DPNH

Als Beispiel für den ablaufenden Dehydrierungs- und Hydrierungsmechanismus sei die Dehydrierung der Milchsäure mit DPN ${ }^{+}$bei Gegenwart des Enzyms LDH angeführt:

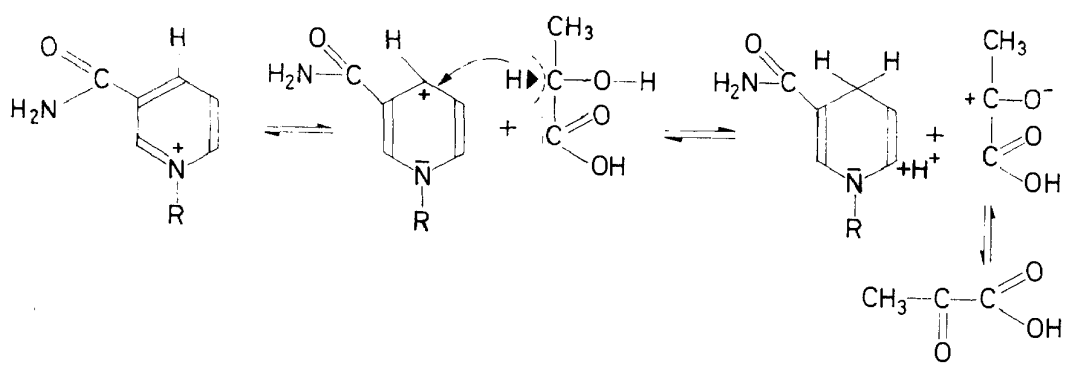

Das $\mathrm{DPN}^{+}$reagiert in seiner mesomeren Form, die die positive Ladung in 4-Stellung trägt. Der Wasserstoff, der auf den Pyridinring übertragen wird, lagert sich mit seinem Elektronenpaar, also als Hydrid-Ion, an. Die Reaktion verläuft außerdem stereospezifisch, worauf aber nicht näher eingegangen werden soll.

Die physikalische Grundlage des optischen Tests bildet das LambertBeer'sche Gesetz:

$$
\log I_{0} / I=E=\varepsilon \cdot c \cdot d
$$

$\left[I^{\circ}=\right.$ Lichtintensität des eintretenden Lichtstrahls; $I=$ Lichtintensität des austretenden Lichtstrahls; $\varepsilon=$ molarer Extinktionskoeffizient; $c=$ Konzentration in Molen $/ \mathrm{ml} ; d=$ Schichtdicke.]

Das Lambert-Beer'sche Gesetz besagt, daß die gemessene Extinktion linear proportional der Konzentration des absorbierenden Stoffes ist. Jede Eichkurve, die man dadurch erhält, daß man die gemessene Extinktion gegen die eingesetzte Konzentration aufträgt, ist eine Gerade. Voraussetzung für die Anwendung des Lambert-Beer'schen Gesetzes ist, daß man mit monochromatischem Licht arbeitet. 


\section{O. BALGKE}

Wenden wir nun das Lambert-Beer'sche Gesetz auf die Lichtabsorption des Überganges $\mathrm{DPN}^{+} \rightleftharpoons \mathrm{DPNH}$ an.

Der molare Extinktionskoeffizient $\varepsilon$ für DPNH beträgt bei $340 \mathrm{~m} \mu$ 6,22: $10^{6}$ $\mathrm{cm}^{2} / \mathrm{Mol}$ und bei $366 \mathrm{~m} \mu\left(25^{\circ} \mathrm{C}\right) 3,3 \cdot 10^{6} \mathrm{~cm}^{2} / \mathrm{Mol}$. Daraus folgt: $1 \mu \mathrm{Mol}=$ $663,3 \mu \mathrm{g}$ DPN + in I ml gelöst, schwächt nach der enzymatischen Reduktion zu DPNH das Licht der Wellenlänge $340 \mathrm{~m} \mu$ bei einer Schichtdicke von 1 $\mathrm{cm}$ um den $\log I_{0} / I=6,22$, bei $366 \mathrm{~m} \mu$ um $\log I_{0} / I=3,3$.

Wenn man also die Extinktion log $I_{0} / I$ des DPNH bei 340 oder $366 \mathrm{~m} \mu$ mißt, so kann man den DPNH-Gehalt in der Küvette berechnen. Läßt man z.B. Milchsäure mit $\mathrm{DPN}^{+}$bei Gegenwart der Lactat-Dehydrogenase reagieren, so wird Milchsäure zu Brenztraubensäure dehydriert. Hierbei entsteht die äquivalente Menge an DPNH. Folglich kann man auf Grund der gemessenen DPNH-Absorption bei 340 bzw. $366 \mathrm{~m} \mu$ ermitteln, wieviel Milchsäure zu Brenztraubensäure dehydriert wurde.

Umgekehrt kann man natürlich auch die Hydrierung der Brenztraubensäure zu Milchsäure im optischen Test verfolgen. Da hierbei DPNH verbraucht wird, muß die Absorption des DPNH abnehmen.

Man kann also die Zunahme und Abnahme an DPNH in enzymatischen Reaktionen messen. Diese Konzentrationsänderungen des DPNH entsprechen den molaren Konzentrationen an dehydriertem oder hydriertem Substrat.

Ich möchte noch kurz darauf hinweisen, daß das System Triphosphopyridin-nucleotid $\left(\mathrm{TPN}^{+}\right) \rightleftharpoons$ Dihydrotriphospho-pyridin-nucleotid (TPNH), welches bei einigen Dehydrogenasen als Coenzym auftritt, die gleichen optischen Eigenschaften aufweist wie das DPN ${ }^{+} \rightleftharpoons$ DPNH-System.

$\mathrm{Da}$ wir die enzymatische Reaktion in der Küvette im optischen Test ablaufen lassen, können wir auch die Geschwindigkeit der Reaktion messen, nämlich die Geschwindigkeit, mit der sich DPNH bildet oder verbraucht wird. Bekanntlich definiert man die Reaktionsgeschwindigkeit als Konzentrationsänderung pro Zeiteinheit. Wie wir eben gesehen haben, ist die Extinktionsänderung proportional der Konzentrationsänderung. Man kann also im optischen Test die Reaktiongsgeschwindigkeit bestimmen.

Nun ist die Geschwindigkeit einer enzymatischen Reaktion durch die Enzymmenge bedingt, die die Reaktion mehr oder weniger katalysiert. Man kann folglich aus der Geschwindigkeit einer Enzym-katalysierten Reaktion auf die Menge bzw. auf die katalytische Aktivität des Enzyms schließen.

Wir können daher zwei Gruppen der enzymatischen optischen Tests unterscheiden:

(1) Bestimmung von Konzentrationen physiologischer Substanzen, wie Ketosäuren, Coenzyme, z.B. DPN ${ }^{+}$bzw. DPNH selbst, Adenosintriphosphat, usw.;

(2) Bestimmung von Enzymaktivitäten.

Im ersten Falle bestimmen wir die Konzentration auf Grund der Zu- oder Abnahme des DPNH, indem wir die gesamte Extinktionszu- oder -abnahme bei 340 bzw. $366 \mathrm{~m} \mu$ messen.

Im zweiten Falle ermitteln wir die Reaktionsgeschwindigkeit, also die Abnahme der Konzentration pro Zeiteinhelt, indem wir die Extinktionsänderung pro Zeiteınheit messen. 
Im $A b b .2$ sehen Sie die graphische Darstellung eines Versuchsprotokolls zu einer Substratbestimmung im Serum, z.B. der Brenztraubensäure. Bei der praktischen Durchführung dieses Tests muß zunächst das Serum enteiweißt werden, damit die serumeigene Lactat-Dehydrogenase zerstört wird. Nach Zusatz von DPNH kann die Brenztraubensäure des Serums nun nicht mehr hydriert werden, weil eben das spezifische Enzym LDH fehlt. Durch Zugabe eines Überschusses von LDH wird die Reaktion gestartet. Jetzt reagiert die Brenztraubensäure mit dem DPNH zu Milchsäure und das DPNH wird zu DPN+ dehydriert. Die DPNH-Absorption nimmt im gleichen Verhältnis $a b$, wie Brenztraubensäure vorgelegen hat. Die Reaktion steht nach wenigen Minuten und es wird die gesamte Extinktionsänderung gemessen.

Bei einer solchen Endwert-Bestimmung beobachtet man häufig, daß die Reaktion - aus nicht immer erklärlichen Grunden - nicht völlig zum Stillstand kommt. Sie sehen in $\operatorname{der} A b b .2$, daß die Extinktion langsam

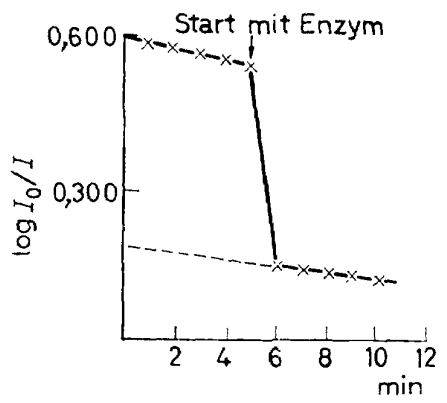

$A b b$. 2. Graphische Darstellung eines Versuchsprotokolls zu einer Substratbestimmung im Serum. (Messgröße: $\Delta \mathrm{E}$.)

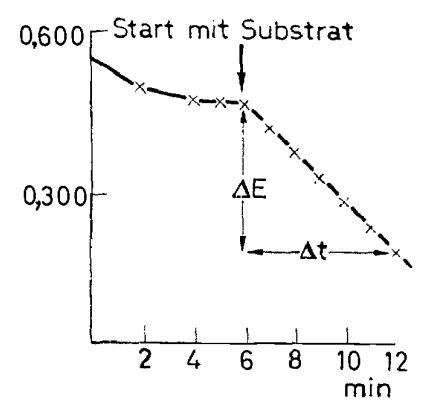

$A b b$. 3. Graphische Darstellung einer Enzymaktivitäts-Bestimmung. (Messgrößen: $\Delta \mathrm{E} / \Delta \mathrm{t}$.)

abfällt ("schleicht"). Hier muß man zur exakten Bestimmung des Endwertes, falls die jeweilige Fragestellung es erfordert, auf die Zeit $t_{0}$ extrapolieren.

Nach dem gleichen Prinzip, nur daß man hier DPN + in den Test einsetzt, verläuft z.B. die enzymatische Dehydrierungs-Reaktion von Äthylalkohol mit DPN+ bei Gegenwart des Enzyms Alkohol-Dehydrogenase. Diese Reaktion liegt der enzymatischen Alkoholbestimmung, der sog. ADHMethode, zugrunde. In dieser Dehydrierungs-Reaktion wird DPN + zu DPNH hydriert, und man mißt die Zunahme an DPNH, die proportional der vorhandenen Alkoholmenge ist.

Im $A b b .3$ sehen Sie die graphische Darstellung einer EnzymaktivitätsBestimmung, z.B. im Serum. Da auch andere Enzyme im Serum vorhanden sind, deren Aktivität wir nicht bestimmen wollen, lassen wir diese nach Zugabe von DPNH-Überschuß mit den serumeigenen Substraten abreagieren (z.B. die LDH-Reaktion mit der im Serum vorhandenen Brenztraubensäure). Dies erkennt man in der $A b b .3$ an dem Extinktionsabfall vor dem Start der Reaktion. Nachdem die Reaktion steht, startet 


\section{O. BALCKE}

man die zu messende Enzym-Reaktion z.B. mit Ketoglutarsäure, wenn man die Serum-Transaminasen bestimmen will. Gremessen wird die Extinktionsänderung pro Minute, d.h. die Geschwindigkeit der in der Küvette ablaufenden Reaktion.

Hinsichtlich der praktischen Durchführung der optischen Tests besteht der Unterschied zwischen der Bestimmung von Enzymaktivitäten und Substratkonzentrationen, z.B. im Serum darin, daß man bei der AktivitätsBestimmung nicht enteiweißt und die Reaktion mit Substrat startet, und daß man bei der Bestimmung von Substratkonzentrationen enteiweißen muß und die Reaktion mit Enzym startet.

Die enzymatische Bestimmung von Intermediär-Produkten mit Hilfe DPN +-abhängiger Dehydrogenasen braucht sich nun nicht auf Substanzen zu beschränken, die direkt in einer Dehydrogenase-Reaktion umgesetzt werden. Man kann in sehr vielen Fällen eine solche DehydrogenasenReaktion als "Indikator-Reaktion" anhängen.

Als Beispiel eines solchen zusammengesetzten optischen Tests betrachten wir die Bestimmung des Substrats Phospho-enol-brenztraubensäure (PEP). Bei Gegenwart des Enzyms Pyruvatkinase setzt sich die PEP mit Adenosindiphosphat (ADP) zu Brenztraubensäure und Adenosintriphosphat (ATP) um. Die gebildete Brenztraubensäure ist das Substrat einer DPN+abhängigen Dehydrogenase, der LDH. Wenn man also dem Testansatz LDH und DPNH zusetzt, so wird die aus der PEP gebildete Brenztraubensäure in der angehängten "Indikator-Reaktion" von dem vorhandenen DPNH zu Milchsäure hydriert:
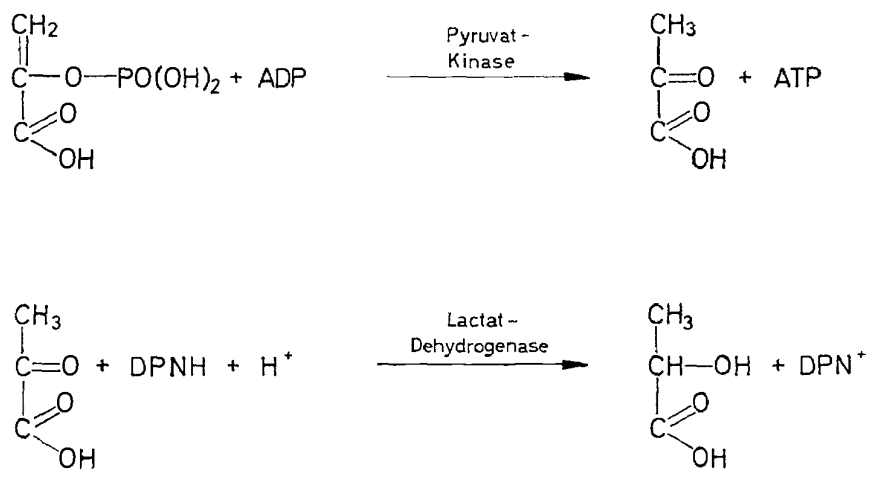

Die gemessene Abnahme der DPNH-Absorption entspricht genau der vorhandenen Konzentration an Phospho-enol-brenztraubensäure.

Man kann auch ohne weiteres einen dreifach zusammengesetzten optischen Test durchführen, z.B. die Bestimmung von Creatin:

$\begin{array}{lll}\text { Creatin + ATP } & \begin{array}{c}\text { Creatin-Phospho- } \\ \text { kinase }\end{array} & \text { Creatinphosphat + ADP } \\ \text { PEP + ADP } & \stackrel{\text { Pyruvatkinase }}{\rightleftharpoons} & \text { Pyruvat + ATP } \\ \text { Pyruvat + DPNH }+\mathrm{H}+\stackrel{\mathrm{LDH}}{\rightleftharpoons} & \text { Lactat }+ \text { DPN }+\end{array}$




\section{DIE THEORIE DES OPTISGHEN TESTS NAGH O. WARBURG}

Das eben geschilderte Prinzip des zusammengesetzten optischen Tests läßt sich natürlich auch auf die Bestimmung von Enzymaktivitäten anwenden. Ein Beispiel aus der Praxis, der SGOT-Test, mag dies veranschaulichen:<smiles>NC(CC(=O)O)C(=O)O</smiles>

L-Asparaginsäure

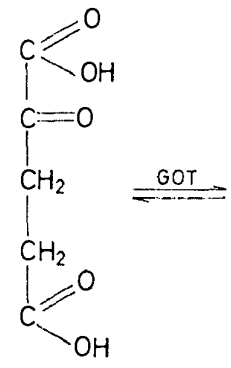

$\alpha$-Ketoglutarsäure<smiles>O=C(O)CC(=O)O</smiles>

Oxalessigsäure

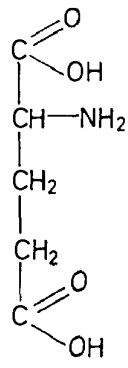

L-Glutaminsäure<smiles>O=C(O)CC(=O)C(=O)O</smiles><smiles>O=C(O)CC(O)C(=O)O</smiles>

$+\mathrm{DPN}^{+}$

Bei der Bestimmung der Glutamat-Oxalacetat-Transaminase im Serum wird die Geschwindigkeit gemessen mit der Asparaginsäure und $\alpha$-Ketoglutarsäure umgesetzt werden in Oxalessigsäure und Glutaminsäure. Das Reaktionsprodukt Oxalessigsäure ist nun in der "Indikator-Reaktion" mit DPNH das Substrat des "Indikator"-Enzyms Malat-Dehydrogenase. Bei der praktischen Durchführung eines solchen zusammengesetzten Tests ist nun besonders darauf zu achten, daß die Geschwindigkeit der IndikatorReaktion sehr schnell ist gegenüber der Geschwindigkeit der zu messenden Reaktion. Denn es soll ja ermittelt werden, wie schnell die Transaminase Oxalessigsäure anliefert, und dies kann man nur messen, wenn praktisch im selben Augenblick die entstehende Oxalessigsäure sich mit DPNH umsetzt, d.h. wenn im gleichen Augenblick der Entstehung von Oxalessigsäure sich entsprechend die DPNH-Konzentration und damit die Lichtabsorption ändert.

In unserem Beispiel ist die Substratkonzentration sehr klein, weil die gebildete Oxalessigsäure sofort weiter reagiert. Wenn man die "IndikatorReaktion "schneller gestalten muß, so kann man dies nur dadurch erreichen, indem man die Konzentration des "Indikator"-Enzyms erhöht. Denn bei Konstanz der Substratkonzentration ist die Reaktionsgeschwindigkeit proportional der Enzymkonzentration. Man kann diese Überlegungen auf Grund der von Michaelis und Menten aufgestellten Postulate über die Geschwindigkeitsverhältnisse bei der Bildung bzw. bei dem Zerfall eines 


\section{O. BALCKE}

Enzym-Substrat-Komplexes ableiten, die hier aber nicht näher erörtert werden sollen.

Zum Abschluß möchte ich noch kurz erwähnen, daß man bei der Durchführung eines enzymatischen optischen Tests auf einige besondere Bedingungen der Enzymaktivität zu achten hat.

Da die Enzyme Eiweißstoffe sind, ist natürlich der pH-Wert des Reaktionsmediums für den Ablauf einer enzymatischen Reaktion sehr wichtig. Die meisten Enzyme entfalten ihre spezifische katalytische Wirkung nur innerhalb eines bestimmten $\mathrm{pH}$-Bereiches, dan man als das $\mathrm{pH}$-Optimum des betreffenden Enzyms bezeichnet. Wie bei allen anderen chemischen Reaktionen werden natürlich auch die Reaktionsgeschwindigkeiten der enzymatischen Reaktionen von der Temperatur beeinflußt. Eine Temperaturerhöhung um $10^{\circ}$ kann eine Steigerung der Reaktionsgeschwindigkeit um das zwei- bis vierfache bewirken. Allerdings sind diesem Effekt auf Grund der Temperaturempfindlichkeit der Eiweißstoffe rasch Grenzen gesetzt.

Schließlich sei noch erwähnt, daß viele Enzyme Ionen als Aktivatoren benötigen, andererseits auch manche Ionen einen Inhibitoreffekt auf die enzymatischen Reaktionen ausüben.

\section{Literaturverzeichnis}

G. Beisenherz, H. J. Boltze, T. Bücher, R. Czok, K. H. Garbade, E. Meyer-Arendt u. G. Pfleiderer. Z. Naturforsch., 8b, 555 (1953)

R. K. Bonnichsen. Acta Chem. Scand., 4, 714 (1950)

H. von Euler. Chemie d. Enzyme, Band II, p. 3, J. F. Bergmann, München (1934)

D. E. Green u. H. Beinert. Am. Rev. Biochem., 24, 1 (1955)

A. Harden u. W. J. Young. Proc. Roy. Soc. (London), B77, 405 (1906)

B. Heß. Klin. Wochschr., 33, 540 (1955)

H. J. Hohorst. Biochem. Z., 328, 509 (1956)

B. L. Horecker u. A. Kornberg. F. Biol. Chem., 175, 385 (1948)

N. O. Kaplan. Record Chem. Progr. (Kresge-Hooker Sci. Lib.), 16, 177 (1955)

N. O. Kaplan, M. M. Ciotti, F. E. Stolzenbach u. N. R. Bacur. F. Am. Chem. Soc., 77, 815 (1955)

W. Lamprecht. Dissertation, Techn. Hochschüle, München (1954)

F. A. Loewus, B. Venneslaud u. D. L. Harris. F. Am. Chem. Soc., 77, 3391 (1955)

E. Negelein. Unveröffentlicht (Protokoll u. Manuskript durch Kriegseinwirkung verloren)

E. Negelein u. E. Haas. Biochem. Z., 282, 206 (1935)

P. Ohlmeyer. Biochem. Z., 297, 66 (1938)

M. E. Pullmann, A. San Pietro u. S. P. Colowick. F. Biol. Chem., 206, 129 (1954)

E. Racker. F. Biol. Chem., 184, 313 (1950)

G. W. Rafter u. S. P. Colowick. F. Biol. Chem., 209, 773 (1954)

F. Schlenk u. H. von Euler. Naturwissenschaften, 24, 794 (1936)

D. Stewart u. S. Gutcho. Anal. Chem., 20, 1185 (1948)

W. Thorn, G. Pfleiderer, R. A. Frowein u. G. Ross. Arch. ges. Physiol. Pflüger's, 261, 334 (1955)

B. Vennesland. F. Cellular Comp. Physiol., 47, Suppl. 1, 201 (1956)

K. Wallenfels u. W. Christian. Angere. Chem., 64, 419 (1952)

O. Warburg, W. Christian u. A. Griese. Biochem. Z., 282, 157 (1935)

O. Warburg. Die wasserstofübertragenden Fermente, Springer Verlag, Berlin (1944) 